TRANSACTION OF THE

AMERICAN MATHEMATICAL SOCIETY

Volume 177, March 1973

\title{
PAIRINGS AND PRODUCTS IN THE HOMOTOPY SPECTRAL SEQUENCE
}

BY

\author{
A. K. BOUSFIELD AND D. M. $\operatorname{KAN}\left({ }^{1}\right)$
}

\begin{abstract}
Smash and composition pairings, as well as Whitehead products are constructed in the unstable Adams spectral sequence; and the se pairings and products are described homologically on the $E_{2}$ level. In the special case of the Massey-Peterson spectral sequence, the composition action is given homologically by the Yoneda product, while the Whitehead product vanishes. It is also shown that the unstable Adams spectral sequence over the rationals, with its Whitehead products, is given by the primitive elements in the rational cobar spectral sequence.
\end{abstract}

1. Introduction. The purpose of this paper is to show that the homotopy spectral sequence $E_{r}(X ; R)$ of a space $X$ (with base point) with coefficients in a ring $R$, which we defined in [7], admits smash and composition pairings as well as Whitebead products. The paper is divided into four chapters.

Chapter $I$ is introductory. In it we associate with a cosimplicial space $\mathbf{Y}$ a tower of fibrations and hence a spectral sequence $E_{r} Y$, in such a manner that $E_{r}(X ; R)=E_{r} \mathbf{R} X$, where $\mathbf{R} X$ denotes the cosimplicial space obtained by "resolving $X$ with respect to $R$."

In Chapter II we construct the smash and composition pairings. For this we first observe that, for any two cosimplicial spaces $\mathbf{X}$ and $\mathbf{Y}$, there exists a basic pairing of spectral sequences $E_{r} \mathbf{X} \otimes E_{r} \mathbf{Y} \rightarrow E_{r}(\mathbf{X} \wedge \mathbf{Y})$. This is rather unpleasant to prove in our present setting (i.e. using towers of fibrations), but not, as we show in [8], if one approaches the spectral sequence of a cosimplicial space in a different (but of course equivalent) way. And we then obtain the desired smash and composition pairings by composing this basic pairing for suitable $\mathbf{X}$ and $\mathbf{Y}$ with appropriate spectral sequence maps.

In Chapter III we construct the Whitehead product by first constructing a Samelson product for the loops and then delooping. To do this we need an analogous

Received by the editors February 29, 1972.

AMS (MOS) subject classifications (1970). Primary 55H15, 55J10, 55H05; Secondary $18 \mathrm{G} 10,55 \mathrm{E} 99$.

Key words and phrases. Unstable Adams spectral sequence, simplicial set, cosimplicial resolution, composition pairing, Whitehead product.

(1) This research was partially supported by NSF GP 8885 and NSF GP 23122. 
basic pairing of spectral sequences for cosimplicial simplicial groups, for which we again refer the reader to [8].

Chapter IV contains homological descriptions of our pairings and products on the $E_{2}$-level for the important case $R=Z_{p}$, the integers modulo a prime $p$, as well as for $R=Q$, the rationals. Indeed for $R=Q$ we show that our spectral sequence can be obtained by merely taking primitive elements in the rational cobar spectral sequence [1].

\section{CHAPTER I. THE HOMOTOPY SPECTRAL SEQUENCE OF A COSIMPLICIAL SPACE}

2. Cosimplicial objects. We start with recalling from [7] the notion of an (augmented) cosimplicial object and mentioning our prime example, the resolution of a space with respect to a ring.

2.1. Cosimplicial objects. A cosimplicial object $\mathbf{X}$ (over a category $\mathcal{C}$ ) consists of

(i) for every integer $n \geq 0$ an object $X^{n} \in \mathcal{C}$,

(ii) for every pair of integers $(i, n)$ with $0 \leq i \leq n$ coface and codegeneracy maps

$$
d^{i}: \mathbf{X}^{n-1} \rightarrow \mathbf{X}^{n} \in \mathcal{C}, \quad s^{i}: \mathbf{X}^{n+1} \rightarrow \mathbf{X}^{n} \in \mathcal{C}
$$

satisfying the identities

$$
\begin{aligned}
d^{j} d^{i} & =d^{i} d^{j-1} & & \text { for } i<j, \\
s^{j} d^{i} & =d^{i} s^{j-1} & & \text { for } i<j, \\
& =\text { id } & & \text { for } i=j, j+1, \\
& =d^{i-1} s^{j} & & \text { for } i>j+1, \\
s^{j} s^{i} & =s^{i-1} s^{j} & & \text { for } i>j .
\end{aligned}
$$

A cosimplicial map $f: X \rightarrow Y$ consists of maps $f: X^{n} \rightarrow Y^{n} \in \mathcal{C}$ which commute with all the cofaces and codegeneracies. $\Lambda$ cosimplicial object (map) over $\mathcal{C}$ thus corresponds to a simplicial object (map) over the dual category $\mathcal{C}^{*}$.

2.2. Augmentations. An augmentation of a cosimplicial object $X$ (over $\mathcal{C}$ ) consists of a map $d^{0}: X^{-1} \rightarrow X^{0} \in \mathcal{C}$ such that $d^{1} d^{0}=d^{0} d^{0}: X^{-1} \rightarrow X^{1}$. We now turn to our prime example.

2.3. The resolution of a space with respect to a ring. Let $\mathfrak{S}_{*}$ denote the category of "spaces", i.e. simplicial sets with base point *. Let $R$ be a ring (with unit), let the "free $R$-module functor" $R: \oint_{*} \rightarrow \oint_{*}$ and the natural transformations $\phi:$ Id $\rightarrow R, \psi: R R \rightarrow R$ be as in [7, \$2] and let $R^{n}=R \cdots R$ ( $n$ copies of $R$ ). For $X \in \mathcal{S}_{*}$ the resolution of $X$ with respect to $R$ then is the augmented cosimplicial object $\mathbf{R} X$ over $\mathcal{S}_{*}$ given by 


$$
\begin{aligned}
& \mathbf{R} X^{n}=R^{n+1} X, \quad n \geq-1, \\
& \mathrm{R} X^{n-1} \stackrel{d^{i}}{\longrightarrow} \mathrm{R} X^{n}=R^{n} X \stackrel{R^{i} \phi R^{n-i}}{\longrightarrow} R^{n+1} X, \\
& \mathrm{R} X^{n+1} \stackrel{s^{i}}{\longrightarrow} \mathrm{R} X^{n}=R^{n+2} \stackrel{R^{i} \psi R^{n-i}}{\longrightarrow} R^{n+1} X .
\end{aligned}
$$

Clearly $\mathbf{R} X$ is natural in $X$ as well as in $R$.

2.4. Remark. In verifying that $\mathbf{R} X$ is indeed an augmented cosimplicial object, one only has to use the fact $(R, \phi, \psi)$ is a triple in the sense of [10]. The same construction thus can be made using other triples.

A way of constructing more cosimplicial objects is by

2.5. Applying a functor. Let $\mathrm{X}$ be an (augmented) cosimplicial object over a category $\mathcal{C}$ and let $T: \mathcal{C} \rightarrow \mathcal{C}^{\prime}$ be a covariant functor. Application of $T$ to $\mathrm{X}$ then yields an (augmented) cosimplicial object $T \mathbf{X}$ over $C^{\prime}$ with $(T \mathbf{X})^{n}=T\left(\mathbf{X}^{n}\right)$ for all $n$.

For instance, if $\mathbf{X}$ is an (augmented) cosimplicial "space", then $\pi_{i} \mathbf{X}(i \geq 2)$ is an (augmented) cosimplicial abelian group.

3. The derivation of a cosimplicial space. In order to define the homotopy spectral sequence of a cosimplicial space in such a manner that it reduces, for $\mathbf{R} X,(2.3)$ to the homotopy spectral sequence of $X$ with coefficients in $R[7, \$ 4]$, we need the derivation construction described below, which generalizes the one of $[7, \S 3]$. First we describe

3.1. A path-like construction. For an (augmented) cosimplicial object $\mathbf{X}$ over $\mathcal{C}$ one can construct a path-like (augmented) cosimplicial object $V \mathbf{X}$ (also over $\mathcal{C}$ ) by lowering the cosimplicial degrees by one and forgetting the first coface and codegeneracy operators, i.e. by setting

$$
\begin{array}{rlrl}
V \mathbf{X}^{n} & =\mathbf{X}^{n+1}, \\
\left(V \mathbf{X}^{n-1} \stackrel{d^{i}}{\longrightarrow} V \mathbf{X}^{n}\right) & =\left(\mathbf{X}^{n} \stackrel{d^{i+1}}{\longrightarrow} \mathbf{X}^{n+1}\right), & 0 \leq i \leq n, \\
\left(V \mathbf{X}^{n+1} \stackrel{s^{i}}{\longrightarrow} V \mathbf{X}^{n}\right) & =\left(\mathbf{X}^{n+2} \stackrel{s^{i+1}}{\longrightarrow} \mathbf{X}^{n+1}\right), & 0 \leq i \leq n .
\end{array}
$$

The objects $\mathbf{X}$ and $V \mathbf{X}$ are related by the cosimplicial map $v: \mathbf{X} \rightarrow V \mathbf{X}$ given by $\left(\mathrm{X}^{n} \stackrel{v}{\rightarrow} V \mathrm{X}^{n}\right)=\left(\mathrm{X}^{n} \stackrel{d^{0}}{\rightarrow} \mathrm{X}^{n+1}\right)$.

Now we can define

3.2. The derivation. Let $\delta_{* K}$ denote the full subcategory of $\mathcal{S}_{*}$ of the Kan complexes with base point and, for $Y \in \mathcal{S}_{* K}$, let $\Lambda Y \stackrel{\lambda}{\rightarrow} Y \in \mathcal{S}_{* K}$ denote the (standard) path fibration over $Y[7, \S 2]$. Let $X$ be an (augmented) cosimplicial space such that $X^{n} \in \mathcal{S}_{* K}$ for $n \geq 0$. Then we define an (augmented) cosimplicial space $D^{1} \mathbf{X}$ (the derivation of $\mathbf{X}$ ) and a cosimplicial map $D^{1} \mathbf{X} \stackrel{\delta}{\rightarrow} \mathbf{X}$ by requiring that $\delta$ is the (cosimplicial) fibre map induced by the map $v: \mathbf{X} \rightarrow V \mathbf{X}$ from the (standard) path fibration $\lambda: \Lambda V \mathbf{X} \rightarrow V \mathbf{X}$, i.e. $\delta$ is given by the pull back diagram 


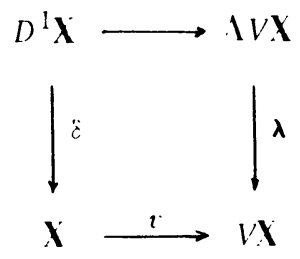

This notion of derivation indeed generalizes the one of $[7, \S 3]$. In fact one readily verifies

3.3. The case $\mathbf{X}=T \mathbf{R} Y$. Let $Y \in \mathcal{S}_{*}$, let $R$ be a ring and let $T: \mathcal{S}_{*} \rightarrow \mathcal{S}_{*}$ be a covariant functor which respects $\mathcal{S}_{* K}[7, \S 3]$. Then one bas the commutative diagram

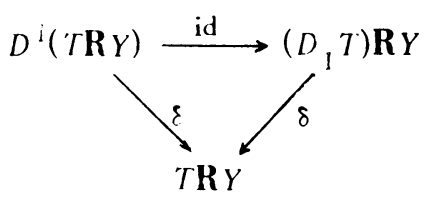

4. The homotopy spectral sequence of a cosimplicial space. In this section we give a definition of the homotopy spectral sequence of an augmented cosimplicial space which directly generalizes $[7, \S 4$ and $\$ 7]$, and discuss some of the immediate consequences of this definition.

4.1. Definition of the spectral sequence. Let $X$ be an augmented cosimplicial space such that $\mathrm{X}^{n} \in \mathcal{S}_{* K}$ for $n \geq 0$. Form the sequence of maps

$$
\cdots \rightarrow D^{s} \mathbf{Y} \stackrel{\delta}{\longrightarrow} D^{s-1} \mathbf{X} \rightarrow \cdots \rightarrow D^{1} \mathbf{X} \stackrel{\delta}{\longrightarrow} D^{0} \mathbf{X}=X
$$

where $D^{i}=D^{1} D^{i-1}$ for all $i \geq 1$, and then define the bomotopy spectral sequence $\left\{E_{r} X\right\}$ of $X$ as the homotopy spectral sequence of the sequence of fibre maps obtained by restricting the above sequence to the augmentations

$$
\cdots \rightarrow D^{s} \mathbf{X}^{-1} \stackrel{\delta}{\longrightarrow} D^{s-1} \mathbf{X}^{-1} \rightarrow \cdots \rightarrow \mathbf{X}^{-1}
$$

"fringed" in dimension 1. By this we mean (as in $[7, \S 4]$ ) that

and that

$$
\begin{aligned}
E_{1}^{s, t} \mathbf{X} & =\pi_{t-s} D^{s} \mathbf{X}^{0}, & & t-1 \geq s \geq 0 \\
& =0, & & \text { otherwise }
\end{aligned}
$$

$$
E_{r}^{s, t} \mathbf{X}=k e r d_{r-1} / i m d_{r-1}, \quad t-1>s \geq 0
$$

but in dimension 1

$$
E_{r}^{s, s+1} \mathbf{X} \subset E_{r}^{s, s+1} \mathbf{X} / i m d_{r-1}, \quad s \geq n
$$

as we define $E_{r}^{s, s+1} \mathrm{X}$ by

$$
E_{r}^{s, s+1} \mathbf{X}=Z_{r-1}^{s, s+1} \mathbf{X} / i m d_{r-1}, \quad s \geq 0
$$


where $Z_{r-1}^{s, s+1} X \subset E_{r-1}^{s, s+1} X$ consists of what "would" have been the cycles, i.e. the elements for which the image under the boundary map $\partial: \pi_{1} D^{s} \mathrm{X}^{0} \rightarrow \pi_{0} D^{s+1} \mathrm{X}^{-1}$ lifts to $\pi_{0} D^{s+r} \mathrm{X}^{-1}$.

One has, of course, to verify that $Z_{r-1}^{s, s+1} \mathrm{X}$ is indeed a group; but this can readily be done as in $[7, \S 4]$. There we also explained why we use a fringe and not an edge.

The above definition generalizes the one of $[7, \$ 4$ and $\$ 7]$. In fact 3.3 implies

4.2. The case of the resolution with respect to a ring. Let $X, \bar{W} \in \mathfrak{S}_{*}$ and let $R$ be a ring. Then, in the notation of $[7, \$ 4$ and $\$ 7]$,

$$
\begin{aligned}
\left\{E_{r}(X ; R)\right\} & =\left\{E_{r} \mathbf{R} X\right\}, \\
\left\{E_{r}(W, X ; R)\right\} & =\left\{E_{r} \operatorname{bom}(W, \mathbf{R} X)\right\} .
\end{aligned}
$$

We can avoid the restriction that $X^{n} \in \mathcal{S}_{* K}$ for $n \geq 0$ by making

4.3. A slight generalization. As [13] there is a natural isomorphism $\left\{E_{r} X\right\} \approx$ $\left\{E_{r} \operatorname{Sin}|\mathbf{X}|\right\}$ where $\|$ and $\operatorname{Sin}$ are the realization and the singular functor, we can and will, whenever $\mathrm{X}^{n}$ is not in $\mathcal{S}_{* K}$ for all $n \geq 0$, define the homotopy spectral sequence $\left\{E_{r} \mathbf{X}\right\}$ of $\mathbf{X}$ by $\left\{E_{r} \mathbf{X}\right\}=\left\{E_{r} \operatorname{Sin}|X|\right\}$.

As in $[7, \$ 4]$ we have the following

4.4. Trivialities about $E_{r} X$ and $E_{\infty} X$.

(i) $d_{r}: E_{r}^{s, t} \mathbf{X} \rightarrow E_{r}^{s+r, t+r-1} \mathbf{X}$;

(ii) $E_{r+1}^{s, t} \mathbf{X} \subset E_{r}^{s, t} \mathbf{X}$, for $r>s$;

(iii) $E_{\infty}^{s, t} \mathbf{X}=\bigcap_{r>s}^{r} E_{r}^{s, t} \mathbf{X}$;

(iv) for $t-1 \geq s \geq 0$ there is a natural short exact sequence

$$
0 \rightarrow\left(F^{s} / F^{s+1}\right)_{t-s} \mathbf{X}^{-1} \stackrel{e_{s}}{\longrightarrow} E_{\infty}^{s, t} \mathbf{X} \rightarrow F^{\infty} \pi_{t-s-1} D^{s+1} \mathbf{X}^{-1} \cap \text { ker } \delta_{*} \rightarrow 0,
$$

where $F^{u} \pi_{q} D^{s} \mathbf{X}^{-1}=i m\left(\pi_{q} D^{s+u} \mathbf{X}^{-1} \rightarrow \pi_{q} D^{s} \mathbf{X}^{-1}\right)$ and $F^{\infty} \pi_{q} D^{s} \mathbf{X}^{-1}=\bigcap_{u} F^{u} \pi_{q} D^{s} \mathbf{X}^{-1}$.

Finally we observe

4.5. The nonrole of the augmentation. The spectral sequence does not really depend on the augmentation, i.e. if $\mathbf{X}$ is an augmented cosimplicial space and $\mathbf{Y} \subset$ $\mathbf{X}$ is sucb that $\mathbf{Y}^{n}=\mathbf{X}^{n}$ for $n \geq 0$ and $\mathbf{Y}^{-1}=*$, then the inclusion $\mathbf{Y} \rightarrow \mathbf{X}$ induces isomorphisms $E_{r} \mathbf{Y} \approx E_{r} \mathbf{X}, 1 \leq r \leq \infty$.

This follows readily from the fact that the tower $\left\{D^{s} Y^{-1}, \delta\right\}$ used to define $E_{r} \mathbf{Y}$ is induced by the map $\mathrm{Y}^{-\mathrm{i}} \rightarrow \mathrm{X}^{-1}$ from the tower $\left\{D^{s} \mathrm{X}^{-1}, \delta\right\}$ used to define $E_{r} X$.

4.6. A homology analogue. It was pointed out by D. L. Rector that one can obtain a bomology spectral sequence by replacing induced fibre maps by induced cofibrations and homotopy groups by homology groups. In [17] he shows that this approach can be used to obtain the Eilenberg-Moore spectral sequence and to introduce therein the Steenrod operations. 
5. A more convenient description of the $E_{1}$-term. We end this chapter with observing that the spectral sequence of a cosimplicial space $X$, as defined in $\$ 4$, has an $E_{1}$-term which is rather inconvenient for constructing pairings and products or studying $E_{2} X$. However, a more convenient description of $E_{1} X$ is possible thanks to the fact, described below, that the groups $\pi_{t-s} D^{s} \mathrm{X}^{0}$ are naturally isomorphic to certain subgroups $\pi_{t}^{\prime} \mathbf{X}^{s} \subset \pi_{t} \mathbf{X}^{s}$, which will be called

5.1. The normalized homotopy groups. For a cosimplicial space $\mathbf{X}$ its normalized bomotopy groups are the subgroups $\pi_{t}^{\prime} \mathbf{X}^{s} \subset \pi_{t} \mathbf{X}^{s}, t, s \geq 0$, defined by

$$
\pi_{t}^{\prime} \mathbf{X}^{s}=\pi_{t} \mathbf{X}^{s} \cap \operatorname{ker} s^{0} \cap \cdots \cap \operatorname{ker} s^{s-1} \text {. }
$$

A simple calculation then yields

5.2. Proposition. Let $\mathbf{X}$ be a cosimplicial object over $\mathcal{S}_{* K}$. Then the boundary'

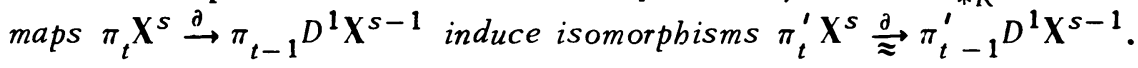

Therefore we can define

5.3. The iterated boundary isomorphism. For a cosimplicial object $\mathbf{X}$ over

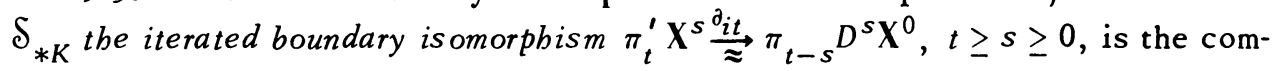
posite isomorphism

$$
\pi_{t}^{\prime} \mathbf{X}^{s} \stackrel{(-1)^{s} \partial}{\longrightarrow} \pi_{t-1}^{\prime} D^{1} \mathbf{X}^{s-1} \stackrel{(-1)^{s-1} \partial}{\longrightarrow} \cdots \stackrel{(-1) \partial}{\longrightarrow} \pi_{t-s}^{\prime} D^{s} \mathbf{X}^{0}=\pi_{t-s} D^{s} \mathbf{X}^{0}
$$

The signs are put in to insure

5.4. Proposition. The following diagram commutes:

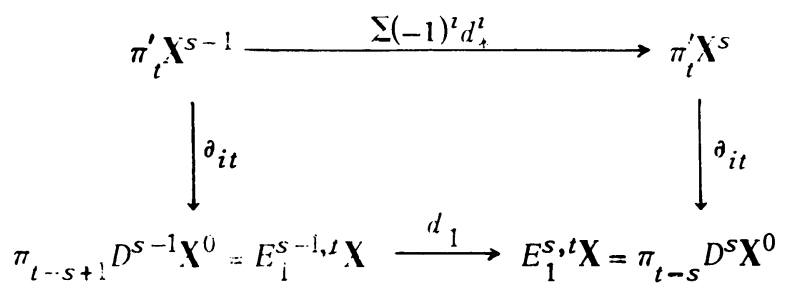

This means that the cosimplicial object $\pi_{*} \mathbf{X}$ contains all the information needed to compute $E_{2} X$. In fact since $\left(\pi_{*}^{\prime} X, \Sigma(-1)^{i} d_{*}^{i}\right)$ is chain equivalent to $\left(\pi_{*} \mathrm{X}, \Sigma(-1)^{i} d_{*}^{i}\right)$ by $\left[12\right.$, p. 236] one needs only the operators $d_{*}^{i}$ on $\pi_{*} \mathrm{X}$ to compute $E_{2} X$ (cf. $[7, \S 10]$ ).

\section{CHAPTER II. SMASH AND COMPOSITION PAIRINGS}

6. The smash and composition pairings of homotopy groups. In this section we recall some "well-known" facts on the smash and composition pairings.

6.1. The smash pairing of homotopy groups. For $X, Y \in \mathcal{S}_{*}$ we denote by

$$
\pi_{t} X \wedge \pi_{t^{\prime}} Y \stackrel{\wedge}{\longrightarrow} \pi_{t+t^{\prime}}(X \wedge Y), \quad t, t^{\prime} \geq 0
$$


the smash pairing $[8, \S 10]$ and recall that this pairing is

(i) linear in the first (second) variable whenever $t>0\left(t^{\prime}>0\right)$,

(ii) associative,

(iii) commutative with sign $(-1)^{t t^{\prime}}$, i.e. $\tau_{*}(u \wedge v)=(-1)^{t t^{\prime}}(v \wedge u)$ for $u \epsilon$ $\pi_{t} X, v \in \pi_{1}, Y$ and $r: X \wedge Y \rightarrow Y \wedge X$ the twisting map.

We consider some special cases.

6.2. The suspension. Let $X \in \mathcal{S}_{*}$, let $S^{1} \in \mathcal{S}_{*}$ be the 1 -sphere, let $S^{m}=S^{1} \wedge$ $\cdots \wedge S^{1} \in \mathcal{S}_{*}$ be the $m$-sphere and let $i$ denote the generator of $\pi_{m} S^{m}$. Then the map

$$
\sigma^{m}=-\wedge i: \pi_{t} X \rightarrow \pi_{t+m}\left(X \wedge s^{m}\right)
$$

clearly is nothing but the m-fold suspension map.

6.3. The composition. Let $W, Y \in \mathcal{S}_{*}$, let bom (, ) denote the "function complex with base point" functor $[7, \S 7]$ and let

$$
\operatorname{bom}(X, Y) \wedge \operatorname{bom}(W, X) \stackrel{c}{\longrightarrow} \operatorname{bom}(W, Y)
$$

be the map which assigns to a pair of $q$-simplices

$$
\Delta[q] \wedge X \stackrel{u}{\longrightarrow} Y, \quad \Delta[q] \wedge W \stackrel{v}{\longrightarrow} X
$$

the composition

$$
\Delta[q] \wedge W \stackrel{\operatorname{diag} \wedge \mathrm{id}}{\longrightarrow} \Delta[q] \wedge \Delta[q] \wedge W \stackrel{\mathrm{id} \wedge v}{\longrightarrow} \Delta[q] \wedge X \stackrel{u}{\longrightarrow} Y .
$$

Then the composition pairing $\circ$ is the composite map

$$
\begin{gathered}
\pi_{t} \operatorname{bom}(X, Y) \wedge \pi_{t^{\prime}} \operatorname{bom}(W, X) \stackrel{\wedge}{\longrightarrow} \pi_{t+t^{\prime}}(\operatorname{bom}(X, Y) \wedge \operatorname{bom}(W, X)) \\
\stackrel{c_{*}}{\longrightarrow} \pi_{t+t^{\prime}} \operatorname{bom}(W, Y) .
\end{gathered}
$$

We end with

6.4. Expressing the smash pairing in terms of the composition pairing. For $X, Y \in \mathfrak{S}_{*}$ the smash pairing

$$
\pi_{t} X \wedge \pi_{t^{\prime}} Y \stackrel{\wedge}{\longrightarrow} \pi_{t+t^{\prime}}(X \wedge Y)
$$

admits a factorization

$$
\pi_{t} X \wedge \pi_{t^{\prime}} Y \stackrel{h_{*} \wedge \mathrm{id}}{\longrightarrow} \pi_{t} \operatorname{bom}(Y, X \wedge Y) \wedge \pi_{t^{\prime}} Y \stackrel{\circ}{\longrightarrow} \pi_{t+t^{\prime}}(X \wedge Y)
$$

where $b: X \rightarrow \operatorname{hom}(Y, X \wedge Y)$ is the map which assigns to a map $u: \Delta[q] \rightarrow X$ (i.e. a $q$-simplex of $X$ ) the map

$$
\Delta[q] \wedge Y \stackrel{u \wedge \mathrm{id}}{\longrightarrow} X \wedge Y .
$$

This follows immediately from the fact that the identity map of $X \wedge Y$ admits a factorization 


$$
X \wedge Y \stackrel{h \wedge \text { id }}{\longrightarrow} \operatorname{bom}(Y, X \wedge Y) \wedge Y \stackrel{c}{\longrightarrow} X \wedge Y .
$$

7. A basic pairing of homotopy spectral sequences. We will derive the smash and composition pairings with coefficients in a ring ( $\$ 8$ and $\$ 9)$ from the following.

7.1. Basic pairing of homotopy spectral sequences. Let $X$ and $Y$ be augmented cosimplicial spaces. Then there exist unique (natural) pairings

$$
E_{r}^{s, t} \mathbf{X} \wedge E_{r}^{s^{\prime}, t^{\prime}} \mathbf{Y} \stackrel{\wedge}{\longrightarrow} E_{r}^{s+s^{\prime}, t+t^{\prime}}(\mathbf{X} \wedge \mathbf{Y}), \quad 1 \leq r \leq \infty,
$$

with the following properties:

(i) the pairing on $E_{1}$ is induced by the iterated boundary isomorphism $\partial_{i t}$ (5.3) from the following pairing of the normalized homotopv groups

$$
\pi_{t^{\prime}}, \mathbf{Y}^{s} \wedge \pi_{t^{\prime}}^{\prime} \mathbf{Y}^{s^{\prime}} \stackrel{f}{\longrightarrow} \pi_{t^{\prime}} \mathbf{X}^{s+s^{\prime}} \wedge \pi_{t^{\prime}}^{\prime} \mathbf{Y}^{s+s^{\prime}} \stackrel{\wedge}{\longrightarrow} \pi_{t+t^{\prime}}^{\prime}(\mathbf{X} \wedge \mathbf{Y})^{s+s^{\prime}},
$$

where $f$ is the (graded) Alexander-Whitney mâp [13, p. 132] given by

$$
f(u, v)=\left((-1)^{t s^{\prime}} d^{s+s^{\prime}} \cdots d^{s+1} u, d^{s-1} \cdots d^{0} v\right) ;
$$

(ii) for $u \in E_{r}^{s, t} \mathbf{X}$ and $v \in E_{r}^{s^{\prime},{ }^{\prime \prime}} \mathbf{Y}(1 \leq r<\infty)$;

$$
d_{r}(u \wedge v)=\left(d_{r} u \wedge v\right)+(-1)^{t-s}\left(u \wedge d_{r} v\right)
$$

(iii) the pairing on $E_{r+1}$ is induced by the one on $E_{r}(1 \leq r<\infty)$ and the pairing on $E_{\infty}$ is induced by the ones on the $E_{r}(1 \leq r<\infty)$;

(iv) the pairing on $E_{\infty}$ is compatible with the smash pairing of the homotopy groups of the augmentations, i.e. if $u \in F^{r} \pi_{t} \mathbf{X}^{-1}$ and $v \in F^{r \prime} \pi_{t^{\prime}} \mathbf{Y}^{-1}$, then $u \wedge v \epsilon$ $F^{r+r^{\prime}} \pi_{t+t^{\prime}}(\mathrm{X} \wedge \mathrm{Y})^{-1}$ and $e_{r+r^{\prime}}(u \wedge v)=e_{r} u \wedge e_{r^{\prime}} v$;

(v) the pairings are bilinear;

(vi) the pairings are associative;

(vii) the pairings are commutative with sign $(-1)^{(t-s)\left(t^{\prime}-s^{\prime}\right)}$ for $r \geq 2$.

Proof. In view of $[8,7.3]$, this is nothing but Theorem 10.4 of [8].

8. The smash pairing with coefficients in a ring. For $X \in \mathcal{S}_{*}$ and $R$ a ring we write (as in $[7, \S 4])\left\{E_{r} X\right\}$ for $\left\{E_{r}(X ; R)\right\}=\left\{E_{r} \mathbf{R} X\right\}$.

Using the basic pairing of $\$ 7$ we now construct

8.1. The smash pairing with coefficients in $R$. For $X, Y \in \mathcal{S}_{*}$ let

$$
R X \wedge R Y \stackrel{a}{\longrightarrow} R(X \wedge Y)=R X \otimes R Y
$$

be the map given by $(x, y) \rightarrow x \otimes y$. The compositions

$$
R^{n+1} X \wedge R^{n+1} Y \stackrel{a}{\longrightarrow} R\left(R^{n} X \wedge R^{n} Y\right) \stackrel{R a}{\longrightarrow} \cdots \stackrel{R^{n} a}{\longrightarrow} R^{n+1}(X \wedge Y)
$$

then yield a cosimplicial map $\mathbf{R} X \wedge \mathbf{R} Y \stackrel{a}{\rightarrow} \mathbf{R}(X \wedge Y)$ and we define this smasb pairing

$$
E_{r}^{s, t} X \otimes E_{r}^{s^{\prime}, t^{\prime}} Y \stackrel{\wedge}{\longrightarrow} E_{r}^{s+s^{\prime}, t+t^{\prime}}(X \wedge Y), \quad 1 \leq r \leq \infty,
$$


as the composition

$$
E_{r} \mathbf{R} \therefore \otimes E_{r} \mathbf{R} Y \stackrel{\wedge}{\longrightarrow} E_{r}(\mathbf{R} X \wedge \mathbf{R} Y) \stackrel{E_{r} a}{\longrightarrow} E_{r} \mathbf{R}(X \wedge Y)
$$

8.2 Properties. The obvious analogues of the properties 7.1(i) through (iv) hold. In particular on $E_{\infty}$ this pairing is compatible with the pairing

$$
\pi_{t} X \wedge \pi_{t^{\prime}} Y \stackrel{\wedge}{\longrightarrow} \pi_{t+t^{\prime}}(X \wedge Y) .
$$

And as the map $a$ is both associative and commutative, properties 7.1(vi) and (vii) also hold.

A special case of this smash pairing is

8.3. The suspension. Let $i \in E_{r}^{0, m} S^{m}(1 \leq r \leq \infty)$ denote the element that corresponds to the generator of $\pi_{m} S^{m}$. Then the smash pairing

restricts to the m-fold suspension

$$
E_{r} X \otimes E_{r} S^{m} \stackrel{\wedge}{\longrightarrow} E_{r}\left(X \wedge S^{m}\right), \quad 1 \leq r \leq \infty,
$$

$$
\sigma_{r}^{m}: E_{r}^{s, t} X \rightarrow E_{r}^{s, t+m}\left(X \wedge S^{m}\right)
$$

given by $u \rightarrow u \wedge i$ for all $u$. This suspension has all the properties implied by 8.2. In particular $\sigma_{\infty}^{m}$ is compatible with the m-fold suspension (6.2) $\sigma^{m}: \pi_{t} X \rightarrow$ $\pi_{t+m}\left(X \wedge S^{m}\right)$.

9. The composition pairing with coefficients in a ring. For $X, W \in \mathcal{S}_{*}$ and $R$ a ring we write (as in $[7, \S 7])\left\{E_{r}(W, X)\right\}$ for $\left\{E_{r}(W, X ; R)\right\}=\left\{E_{r}\right.$ bom $\left.(W, \mathbf{R} X)\right\}$ and construct, again using the basic pairing of $\$ 7$,

9.1. The composition pairing with coefficients in $R$. For $W, X, Y \in \mathfrak{S}_{*}$ the composition pairing

$$
E_{r}(X, Y) \otimes E_{r}(u, X) \stackrel{\circ}{\longrightarrow} E_{r}(U, Y), \quad 1 \leq r \leq \infty,
$$

is defined as the composite map

$$
E_{r} \text { bom }(X, \mathbb{R} Y) \otimes E_{r} \text { bom }(W, \mathbf{R} X) \stackrel{\wedge}{\longrightarrow} \cdots \stackrel{E_{r} c}{\longrightarrow} E_{r} \text { bom }(1 \cdot, \mathbf{R} Y),
$$

where $c$ is the cosimplicial map

$$
b \circ m(\therefore, \mathbf{R} Y) \wedge \text { bom }(w, \mathbf{R} X) \stackrel{c}{\longrightarrow} \operatorname{bom}(w, \mathbf{R} Y)
$$

constructed as follows. For

$$
u: \Delta[q] \wedge H \rightarrow R^{n} X, \quad v: \Delta[q] \wedge X \rightarrow R^{n} Y,
$$

$c(u, v)$ is the composition

$$
\begin{aligned}
\left.\Sigma_{q}\right] \wedge W & \rightarrow \Delta[q] \wedge \Delta[q] \wedge W \rightarrow \Delta[q] \wedge R^{n} X \\
& \rightarrow R^{n}(\triangle[q] \wedge X) \rightarrow R^{2 n} Y \stackrel{w_{n}}{\longrightarrow} R^{n} Y
\end{aligned}
$$


where the unnamed maps are the obvious ones (6.3) and $w_{n}$ is the map which "combines the $i$ th and $(n+i)$ th copies of $R$ ", i.e. $w_{n}$ is the composition

$$
R^{2 n} Y \stackrel{{ }^{t}+1}{\longrightarrow} \because \stackrel{{ }^{t}{ }_{2 n-1}}{\longrightarrow} R^{2 n} Y \stackrel{s^{0}}{\longrightarrow} R^{2 n-1} Y \stackrel{R_{n-1}}{\longrightarrow} R^{n} Y
$$

where $w_{1}=s^{0}$ and $t_{i}: R^{2 n} Y \rightarrow R^{2 n} Y$ is the map which "interchanges the $i$ th and ( $i-1)$ th copies of $R$ (counted from $Y$ )", i.e.

$$
t_{2 n-i}=d^{i} s^{i}+d^{i+1} s^{i}-\mathrm{id} .
$$

The proof that $c$ is indeed a cosimplicial map is straightforward (but not short).

9.2. Properties. Again the obvious analogues of 7.1(i) through (iv) hold. In particular on $E_{\infty}$ the pairing is compatible with the composition pairing (6.3)

$$
\pi_{t} \text { bom }(X, Y) \wedge \pi_{t^{\prime}} \text { bom }(W, X) \stackrel{\circ}{\longrightarrow} \pi_{t+t^{\prime}}(W, Y)
$$

And as the map $c$ is associative (verification of which is lengthy but straightforward), property 7.1(vi) also holds.

We end with

9.3. Expressing the smash pairing in terms of the composition pairing. For $X$, $Y \in \mathcal{S}_{*}$ the smash pairing $E_{r} X \otimes E_{r} Y \stackrel{\wedge}{\rightarrow} E_{r}(X \wedge Y), 1 \leq r \leq \infty$, admits a factorization

$$
E_{r} X \otimes E_{r} Y \stackrel{E_{r} h \otimes \mathrm{id}}{\longrightarrow} E_{r}(Y, X \wedge Y) \otimes E_{r} Y \stackrel{\circ}{\longrightarrow} E_{r}(X \wedge Y)
$$

where $b: \mathbf{R} X \rightarrow \operatorname{bom}(Y, \mathbf{R}(X \wedge Y))$ is the map which assigns to a map $u: \Delta[q] \rightarrow$ $R^{n} X$ the composition

$$
\Delta[q] \wedge Y \stackrel{u \wedge \text { id }}{\longrightarrow} R^{n} X \wedge Y \rightarrow R^{n}(X \wedge Y) .
$$

where the second map is the obvious one.

This follows directly from the commutativity of the diagram

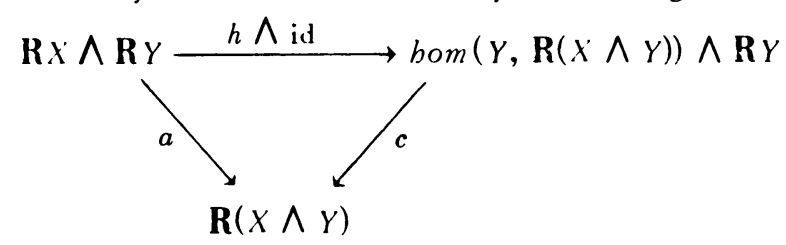

the verification of which is, as usual, lengthy but straightforward.

10. The (composition) action of $E_{r} S^{m}$. Here we discuss how $E_{r} S^{m}$ acts on (most of) $E_{r} X$ by means of composition (for fixed $R$, of course). First a

10.1. Lemma. For $X \in \mathcal{S}_{*}$ the map

$$
E_{r}^{s, t}\left(S^{m}, X\right) \stackrel{r}{\longrightarrow} E_{r}^{s, t+m} X, \quad 1 \leq r \leq \infty
$$

obtained by restricting the composition pairing 


$$
E_{r}^{s, t}\left(S^{m}, X\right) \otimes E_{r}^{0, m} S^{m} \stackrel{\circ}{\longrightarrow} E_{r}^{s, t+m} X
$$

to the generator $i \in E_{r}^{0, m} S^{m}(8.3)$, is an isomorphism if $t-s \geq 1$.

The proof is easy.

Now we can define

10.2. The action of $E_{r} S^{m}$ on $E_{r} X$. The (composition) action of $E_{r} S^{m}$ on $E_{r} X$

$$
E_{r}^{s, t+m} X \otimes E_{r}^{s^{\prime}, t^{\prime}} S^{m} \stackrel{\circ}{\longrightarrow} E_{r}^{s+s^{\prime}, t+t^{\prime}} X, \quad 1 \leq r \leq \infty
$$

is the composition (defined only when $t-s \geq 1$ )

$E_{r}^{s, t+m} X \otimes E_{r}^{s^{\prime}, t^{\prime}} S^{m} \stackrel{r^{-1} \otimes \mathrm{id}}{\longrightarrow} E_{r}^{s, t}\left(S^{m}, X\right) \otimes E_{r}^{s^{\prime}, t^{\prime}} S^{m} \stackrel{\circ}{\longrightarrow} E_{r}^{s+s^{\prime}, t+t^{\prime}} X$.

On $E_{\infty}$ this action is clearly compatible with the unsual action of $\pi_{*} S^{m}$ on $\pi_{*} X, \pi_{t+m} X \otimes \pi_{t^{\prime}} S^{m} \rightarrow \pi_{t+t^{\prime}} X$.

On $X \wedge S^{m}$ this action is closely related to the smash pairing. In fact 9.3 readily implies

10.3. Proposition. The following diagram commutes for all $1 \leq r \leq \infty$ :

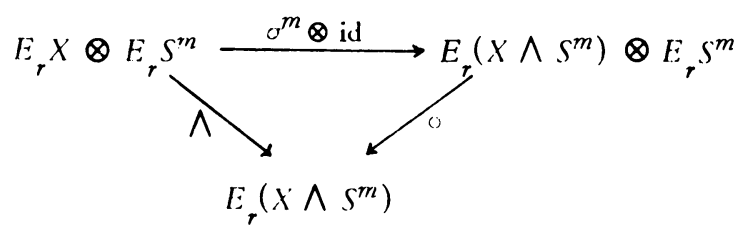

\section{CHAPTER III. WHITEHEAD PRODUCTS}

11. The homotopy spectral sequence of a cosimplicial simplicial group. Let $X \in \mathcal{S}_{*}$ and let $G$ be the loop group functor [13]. Then (up to a possible sign) the Whitebead product in $\pi_{*} X$ corresponds under the boundary isomorphism to the Samelson product in $\pi_{*} G X$. The latter is the more natural notion (it adds dimensions) and handles easier. In order to construct, for a ring $R$, a Whitehead product in $E_{r} \mathbf{R} X$ we will first introduce a Samelson product in $E_{r} G \mathbf{R} X$ and then translate the result to $E_{r} \mathbf{R} X$. Hence we start with explaining what we mean by

11.1. The homotopy spectral sequence of a cosimplicial simplicial group. The approach of $\$ 4$ applied to an augmented cosimplicial simplicial group B causes problems in dimension 0 . To get around this we define $\left\{E_{r} \mathbf{B}\right\}$ by requiring that $\left\{E_{r} \mathbf{B}\right\}$ be isomorphic with $\left\{E_{r} \bar{W} \mathbf{B}\right\}$, where $\bar{W}$ denotes the classifying functor [13]. To be precise we requíre

Let $\partial: \pi_{t+1} \bar{W} \mathbf{B}^{s} \approx \pi_{t} \mathbf{B}^{s}$ be the boundary is omorphism [13] and let

$$
\partial_{0 t}=(-1)^{t} \partial: \pi_{t+1} \bar{W} \mathbf{B}^{s} \approx \pi_{t} \mathbf{B}^{s}
$$


be the "other" boundary isomorphism. Then there exist for all $t \geq s \geq 0$ (i.e. even if $t-s=0$ ) unique isomorphisms

$$
E_{r}^{s, t+1} \bar{W} \mathbf{B} \stackrel{\partial_{0}^{0 t}}{\approx} E_{r}^{s, t} \mathbf{B}, \quad 1 \leq r \leq \infty
$$

such that

(i) the isomorphism on $E_{1}$ is the composition

$\pi_{t+1-s} D^{s} \bar{W} \mathbf{B}^{0}=E_{1}^{s, t+1} \bar{W} \mathbf{B} \stackrel{\partial_{i t}^{-1}}{\longrightarrow} \pi_{t+1}^{\prime} \bar{W} \mathbf{B}^{s} \stackrel{\partial_{0 t}}{\longrightarrow} \pi_{t}^{\prime} \mathbf{B}^{s} \stackrel{\partial_{i t}}{\longrightarrow} E_{1}^{s, t} \mathbf{B}=\pi_{t-s} D^{s} \mathbf{B}^{0}$

(ii) if $u \in E_{r}^{s, t+1} \bar{W} \mathbf{B}$, then $\partial_{0 t} d_{r} u=d_{r} \partial_{0 t} u$,

(iii) the isomorphism on $E_{r+1}$ is induced by the one on $E_{r}(1 \leq r<\infty)$ and the isomorphism on $E_{\infty}$ is induced by the ones on the $E_{r}(1 \leq r<\infty)$,

(iv) $u \in F^{s} \pi_{t+1} \bar{W} \mathbf{B}^{-1}$ if and only if $\partial_{0 t} u \in F^{s} \pi_{t} \mathbf{B}^{-1}$ and in that case $e_{s} \partial_{0 t} u=\partial_{0 t} e_{s} u$.

11.2. Remark. One readily verifies that the above definition coincides in dimensions $\geq 1$ with the one of $\$ 4$, justifying the use of the same notation. This would $n o t$ have been the case if we had used $\partial$ instead of $\partial_{0 t}$.

For later reference we mention an immediate consequence of the above definition.

11.3 The case $\mathbf{B}=G \mathbf{X}$. Let $\mathbf{X}$ be an augmented cosimplicial space, let $G$ be the loop group functor [13] (applied to the component of the base point), let $\partial$ : $\pi_{t+1} \mathbf{X}^{s} \approx \pi_{t} G \mathbf{X}^{s}$ be the boundary isomorphism [13] and let

$$
\partial_{0 t}=(-1)^{t} \partial: \pi_{t+1} \mathbf{X}^{s} \approx \pi_{t} G \mathbf{X}^{s}
$$

again be the "other" boundary isomorphism. Then there exist unique isomorphisms

$$
E_{r}^{s, t+1} \mathbf{X} \stackrel{\partial^{0} t}{\approx} E_{r}^{s, t} G \mathbf{X}, \quad 1 \leq r \leq \infty
$$

which on $E_{1}$ are the compositions

$$
E_{1}^{s, t+1} \mathbf{X} \stackrel{\hat{o}_{i t}^{-1}}{\longrightarrow} \pi_{t+1}^{\prime} \mathbf{X}^{s} \stackrel{\partial_{0 t}}{\longrightarrow} \pi_{t}^{\prime} G \mathbf{Y}^{s} \stackrel{\partial_{i t}}{\longrightarrow} E_{1}^{s, t} G \mathbf{X}
$$

and for which the obvious analogues of 11.1(ii), (iii) and (iv) bold.

12. The Samelson and Whitehead products for homotopy groups. In this section we recall some "well-known" [4] results about Samelson and Whitehead products.

12.1. The Samelson product in $\pi_{*} G X$. For $X \in \mathcal{S}_{*}$ the Samelson product

$$
\pi_{t} G X \wedge \pi_{t^{\prime}} G X \stackrel{[,]}{\longrightarrow} \pi_{t+t^{\prime}} G X, \quad t, t^{\prime} \geq 0,
$$

is the composition 


$$
\pi_{t} G X \wedge \pi_{t^{\prime}} G X \stackrel{\wedge}{\longrightarrow} \pi_{t+t^{\prime}}(G X \wedge G X) \longrightarrow \pi_{t+t^{\prime}} F(G X \wedge G X) \stackrel{c_{*}}{\longrightarrow} \pi_{t+t^{\prime}} G X
$$

where $F$ is Milnor's "free group on" functor [9], the unnamed map is the obvious one and $c$ is the homomorphism which sends each generator $(a, b)$ into the commutator $a b a^{-1} b^{-1}$. It has the properties

(i) it is linear in the first (second) variable whenever $t>0\left(t^{\prime}>0\right)$,

(ii) it is commutative with sign $(-1)^{t t^{\prime}+1}$,

(iii) it satisfies the Jacobi identity with signs $(-1)^{t t^{\prime \prime}},(-1)^{t^{\prime} t}$ and $(-1)^{t^{\prime \prime} t^{\prime}}$ whenever $t, t^{\prime}, t^{\prime \prime}>0$, i.e.

$$
(-1)^{t t^{\prime \prime}}[[u, v], w]+(-1)^{t^{\prime} t}[[v, w], u]+(-1)^{t^{\prime \prime} t^{\prime}}[[w, u], v]=0
$$

for $u \in \pi_{t} G X, v \in \pi_{t^{\prime}} G X, w \in \pi_{t^{\prime \prime}} G X$ and $t, t^{\prime}, t^{\prime \prime}>0$.

Similarly one has

12.2. The Whitehead product in $\pi_{*} X$. For $X \in \mathcal{S}_{*}$ the Whitebead product

$$
\pi_{t} X \wedge \pi_{t^{\prime}} X \stackrel{[,]}{\longrightarrow} \pi_{t+t^{\prime}-1} X, \quad t, t^{\prime} \geq 1
$$

is the composition

$$
\pi_{t} X \wedge \pi_{t^{\prime}} X \stackrel{\partial \wedge \partial}{\longrightarrow} \pi_{t-1} G X \wedge \pi_{t^{\prime}-1} G X \stackrel{[,]}{\longrightarrow} \pi_{t+t^{\prime}-2} G X \stackrel{\partial^{-1}}{\longrightarrow} \pi_{t+t^{\prime}-1} X
$$

or equivalently (11.3)

$$
\pi_{t} X \wedge \pi_{t^{\prime}} X \stackrel{\partial_{0 t} \wedge \partial_{0 t}}{\longrightarrow} \pi_{t-1} G X \wedge \pi_{t^{\prime}-1} G X \stackrel{[,]}{\longrightarrow} \pi_{t+t^{\prime}-2} G X \stackrel{\partial_{0 t}^{-1}}{\longrightarrow} \pi_{t+t^{\prime}-1} X .
$$

Clearly it has the properties of 12.1 with everywhere $t-1, t^{\prime}-1$ and $t^{\prime \prime}-1$ instead of $t, t^{\prime}$ and $t^{\prime \prime}$.

12.3. Remark. The above definition of the Whitehead product differs from the "usual" one by a sign [4].

13. The Samelson and Whitehead products in $E_{1}$. If, in order to construct a Samelson product in $E_{1} G \mathbf{R} X$, one defines a Samelson product in $\pi_{*}^{\prime} G \mathbf{R} X$ in the obvious manner (7.1(i)), then one gets a trivial product. To get around this we make

13.1. Some observations.

(i) The map $c: F(G X \wedge G X) \rightarrow G X$ obviously admits a factorization

$$
F(G X \wedge G X) \stackrel{c^{\prime}}{\longrightarrow} \Gamma_{2} G X \stackrel{\text { incl }}{\longrightarrow} G X
$$

where $\Gamma_{2}$ denotes the commutator subgroup functor.

(ii) There is a commutative diagram 


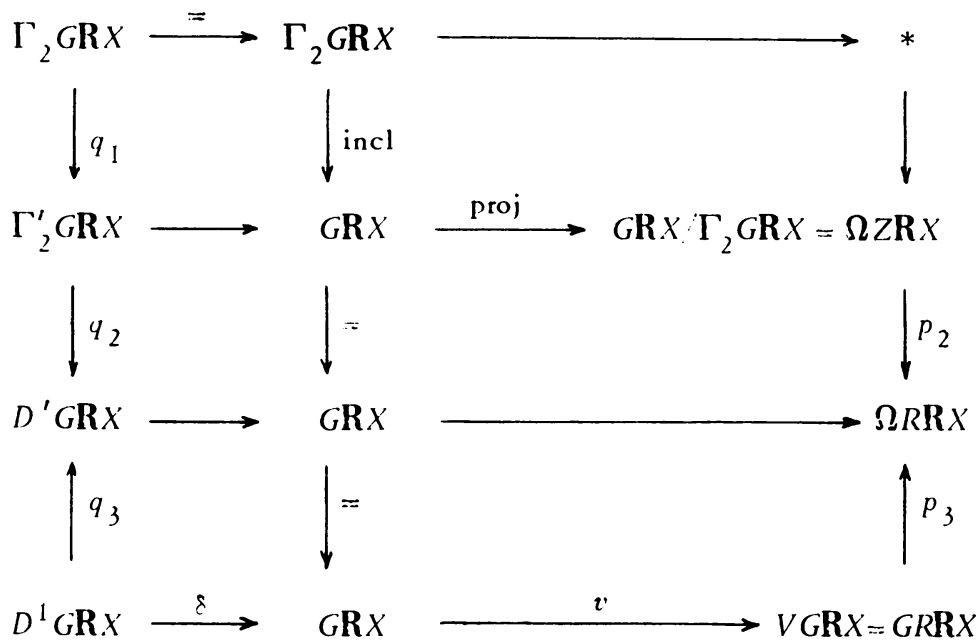

where $\Omega$ denotes the standard loop complex (i.e. the fibre of the standard path fibration $[7, \$ 2]$ ), $p_{2}$ is induced by the ring (with unit) homomorphism $Z \rightarrow R, p_{3}$ sends the generator corresponding to a simplex $y \in R \mathbf{R} X$ into $y-s_{0} d_{0} y$, the horizontal maps on the left are fibre maps induced from a path fibration by the ones on the right, and the vertical maps on the left are induced by the ones on the right.

(iii) As $p_{3}$ induces isomorphisms of the homotopy groups, so does $q_{3}$ and hence composition of $q_{1 *}, q_{2 *}$ and $q_{3}^{-1}$ yields a natural bomomorphism $q_{*}$ : $\pi_{*} \Gamma_{2} G \mathbf{R} X \rightarrow \pi_{*} D^{1} G \mathbf{R} X$.

Now we are ready to construct in a nontrivial manner

13.2. The Samelson product in $\pi_{*}^{\prime} G \mathbf{R} X$. The Samelson product

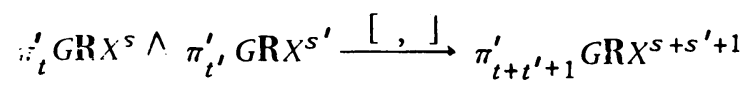

will be the composition (with $f$ as in 7.1 and $\partial$ as in 5.2)

$$
\begin{aligned}
\pi_{t}^{\prime} G \mathbf{R} X^{s} \wedge \pi_{t^{\prime}}^{\prime} G \mathbf{R} X^{s^{\prime}} \stackrel{f}{\longrightarrow} \pi_{t}^{\prime} G \mathbf{R} X^{s+s^{\prime}} \wedge \pi_{t^{\prime}}^{\prime} G \mathbf{R} X^{s+s^{\prime}} \stackrel{\wedge}{\longrightarrow} \pi_{t+t^{\prime}}^{\prime}\left(G \mathbf{R} X^{s+s^{\prime}} \wedge G \mathbf{R} X^{s+s^{\prime}}\right) \\
\longrightarrow \pi_{t+t^{\prime}}^{\prime} F\left(G \mathbf{R} X^{s+s^{\prime}} \wedge G \mathbf{R} X^{s+s^{\prime}}\right) \stackrel{c_{*}^{\prime}}{\longrightarrow} \pi_{t+t^{\prime}}^{\prime} \Gamma_{2} G \mathbf{R} X^{s+s^{\prime}} \\
\stackrel{q_{*}}{\longrightarrow} \pi_{t+t^{\prime}}^{\prime} D^{1} G \mathbf{R} X^{s+s^{\prime}} \stackrel{(-1)^{s+s^{\prime}+\partial^{-1}}}{\longrightarrow} \pi_{t+t^{\prime}+1}^{\prime} G \mathbf{R} X^{s+s^{\prime}+1}
\end{aligned}
$$

And similarly we get

13.3. The Whitehead product in $\pi_{*}^{\prime} R X$. The Whitebead product

$$
\pi_{t}^{\prime} \mathbf{R} X^{s} \wedge \pi_{t^{\prime}}^{\prime} \mathbf{R} X^{s^{\prime}} \stackrel{[,]}{\longrightarrow} \pi_{t+t^{\prime}}^{\prime} \mathrm{R} X^{s+s^{\prime}+1}
$$

will be the composition 


$$
\begin{aligned}
\pi_{t}^{\prime} \mathbf{R} X^{s} \wedge \pi_{t^{\prime}}^{\prime} \mathbf{R} X^{s^{\prime}} & \stackrel{\partial_{0 t} \wedge \partial_{0 t}}{\longrightarrow} \pi_{t-1}^{\prime} G \mathbf{R} X^{s} \wedge \pi_{t^{\prime}-1}^{\prime} G \mathbf{R} X^{s^{\prime}} \\
& \stackrel{[,]}{\longrightarrow} \pi_{t+t^{\prime}-1}^{\prime} G \mathbf{R} X^{s+s^{\prime}+1} \stackrel{\partial_{0 t}^{-1}}{\longrightarrow} \pi_{t+t^{\prime}}^{\prime}, \mathbf{R} X^{s+s^{\prime}+1}
\end{aligned}
$$

For later use $(\$ 19)$ we mention here

13.4. A more direct construction of the Whitehead product in $E_{1}$. For $Y \in \mathcal{S}_{*}$ let $w: R Y \wedge R Y \rightarrow R^{2} Y$ denote the composite map

$$
R Y \wedge R Y \stackrel{\zeta}{\longrightarrow} R(R Y \times R Y) \stackrel{R(+)}{\longrightarrow} R^{2} Y
$$

where, for all $(u, v) \in R Y \wedge R Y$,

$$
\zeta(u, v)=1(u, v)-1(u, *)-1(*, v)
$$

and + denotes the "addition map" $R Y \times R Y \rightarrow R Y$. Then, for $X \in \mathcal{S}_{*}, w$ induces maps

$$
w_{*}: \pi_{*}^{\prime}(\mathbf{R} X \wedge \mathbf{R} X)^{k-1} \rightarrow \pi_{*}^{\prime} \mathbf{R} X^{k},
$$

and a long but straightforward computation shows

13.5. Lemma. The Whitebead product (13.3)

is $(-1)^{t-s-1}$ times the composition

$$
\pi_{t}^{\prime} \mathbf{R} X^{s} \wedge \pi_{t^{\prime}}^{\prime} \mathbf{R} X^{s^{\prime}} \stackrel{[,]}{\longrightarrow} \pi_{t+t^{\prime}}^{\prime} \mathbf{R} X^{s+s^{\prime}+1}
$$

$$
\pi_{t}^{\prime} \mathbf{R} X^{s} \wedge \pi_{t^{\prime}}^{\prime} \mathbf{R} X^{s^{\prime}} \rightarrow \pi_{t+t^{\prime}}^{\prime}(\mathbf{R} X \wedge \mathbf{R} X)^{s+s^{\prime}} \stackrel{w_{*}}{\longrightarrow} \pi_{t+t^{\prime}}^{\prime} \mathbf{R} X^{s+s^{\prime}+1}
$$

where the first map is as in 7.1.

14. The Samelson and Whitehead products in $E_{r}$. Now we use the $E_{1}$-level results of $\$ 13$ to construct a Samelson product in $E_{r} G \mathbf{R} X$ and the desired Whitehead product for $E_{r} \mathbf{R} X$.

14.1. The Samelson product in $E_{r} G \mathbf{R} X$. Let $X \in \mathcal{S}_{*}$ and let $R$ be a ring. Then there exist unique natural products

$$
E_{r}^{s, t} G \mathbf{R} X \wedge E_{r}^{s^{\prime}, t^{\prime}} G \mathbf{R} X \stackrel{[,]}{\longrightarrow} E_{r}^{s+s^{\prime}+1, t+t^{\prime}+1} G \mathbf{R} X, \quad 1 \leq r \leq \infty,
$$

with the following properties.

(i) The product in $E_{1}$ is induced from the Samelson product in $\pi_{*}^{\prime} G \mathbf{R X}(13.2)$ by the iterated boundary isomorphism (5.3).

(ii) For $u \in E_{r}^{s, t} G \mathbf{R} X$ and $v \in E_{r}^{s^{\prime}, t^{\prime}} G \mathbf{R} X(1 \leq r<\infty)$

$$
d_{r}[u, v]=\left[d_{r} u, v\right]+(-1)^{t-s}\left[u, d_{r} v\right] .
$$

(iii) The product in $E_{r+1}$ is induced by the one in $E_{r}(1 \leq r<\infty)$ and the product in $E_{\infty}$ is induced by the ones in the $E_{r}(1 \leq r<\infty)$. 
(iv) The product in $E_{\infty}$ is compatible with the Samelson product in $\pi_{*} G X$,

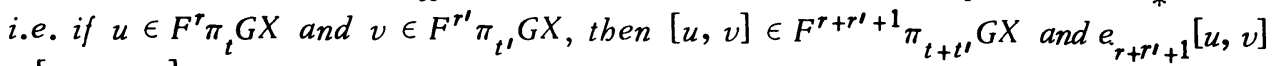
$=\left[e_{r} u, e_{r} p\right]$.

(v) The product is linear in the first (second) variable whenever $t>0\left(t^{\prime}>0\right)$.

(vi) The product is commutative with sign $(-1)^{(t-s)\left(t^{\prime}-s^{\prime}\right)+1}$ for $r>1$.

(vii) The product satisfies the Jacobi identity with signs $(-1)^{(t-s)(t "-s ")}$, $(-1)^{\left(t^{\prime}-s^{\prime}\right)(t-s)}$ and $(-1)^{\left(t^{\prime \prime}-s^{\prime \prime}\right)\left(t^{\prime}-s^{\prime}\right)}$ whenever $t, t^{\prime}, t^{\prime \prime}>0$ and $r>1$.

Proof. Parts (i) through (iv) follow readily from the fact that

(i) the maps $c_{*}^{\prime}, q_{*}$ and $(-1)^{s+s^{\prime}+1} \partial$ of 13.2 induce spectral sequence maps which are compatible with the augmentations, and

(ii) the remaining (composite) map in 13.2 induces (in view of [8, Corollary 4.3 and Theorem 10.8]) a spectral sequence pairing which is also compatible with the augmentations, while (v) through (vii) are consequences of 12.1(i) through (iii) and Theorem 10.8 of [8] or can be proved using [3].

Finally we get, by applying to 14.1 the "other" boundary isomorphism $\partial_{0 t}$ $(\S 11)$,

14.2 The Whitehead product with coefficients in a ring. Let $X \in \mathcal{S}_{*}$ and let $R$ be a ring. Then there exist unique natural products

$$
E_{r}^{s, t} X \wedge E_{r}^{s^{\prime}, t^{\prime}} X .[1,] E_{r}^{s+s^{\prime}+1, t+t^{\prime}} X, \quad 1 \leq r \leq \infty,
$$

such that

(i) the product in $E_{1}$ is induced from the Whitebead product in $\pi_{*}^{\prime} \mathbf{R} X$ (13.3) by the iterated boundary isomorphism (5.3), and

(ii) the obvious analogues of 14.1(ii) through (vii) bold.

\section{CHAPTER IV. APPLICATIONS}

15. The rational spectral sequence $E_{r}(X ; Q)$ and its Whitehead product. As one might expect from the simplicity of rational homotopy theory [16], our rational spectral sequence $E_{r}(X ; Q)$ is already "well known". In fact, we will show below, that our rational spectral sequence $E_{r}(X ; Q)$ (with a Lie algebra structure induced by the Whitehead product) coincides, from $E_{2}$ on, with

(i) the rational version of the lower central series spectral sequence [9]

(with Lie algebra structure induced by the Samelson product), and

(ii) the primitive elements in the rational cobar spectral sequence [1].

This allows us to give a homological description (15.6) of $E_{2}(X ; Q)$.

15.1 The lower central series spectral sequence. Let $X \in \mathcal{S}_{*}$ be connected, let $G$ be the loop group functor [13] and let

$$
\ldots \subset \Gamma_{s+1}^{v} G X \subset \Gamma_{s} G X \subset \ldots \subset \Gamma_{1} G X=G X
$$


be the (integral) lower central series filtration of $G X$ [9]. The associated homotopy exact couple gives rise to the lower central series spectral sequenre $\left\{\hat{E}^{r} X\right\}$ [9] with

$$
\hat{E}_{s, t}^{1} X=\pi,\left(\Gamma_{s} G X / \Gamma_{s+1} G X\right)
$$

and this spectral sequence has a Samelson product $[6, \S 9]$

$$
\hat{E}_{s, t}^{r} X \otimes \hat{E}_{s^{\prime}, t^{\prime}}^{r} X \stackrel{[,]}{\longrightarrow} \hat{E}_{s+s^{\prime} t+t^{\prime}}^{r} X
$$

compatible with the differentials. Now we can state

15.2. Theorem. For $X \in \mathcal{S}_{*}$ connected, the natural spectral sequence map

$$
E_{r}^{s, t+1}(X ; Z)=E_{r}^{s, t+1} \mathbf{Z} X \stackrel{{ }^{\partial} 0 t}{\longrightarrow} E_{r}^{s, t} G \mathbf{Z} X \rightarrow \hat{E}_{s+1, t-s}^{r} X, \quad r \geq 2,
$$

(where $\partial_{0 t}$ is as in $\$ 11$ and the second map is the one described in $[7, \$ 6]$ ) carries Whitebead products in $E_{r}(X ; Z)$ into Samelson products in $E_{r} G \mathbf{Z X}$ and $\hat{E}^{r} X$. Moreover the induced map

$$
E_{r}^{s, t+1}(X ; Q) \approx Q \otimes E_{r}^{s, t+1}(X ; Z) \rightarrow Q \otimes \hat{E}_{s+1, t-s}^{r} X, \quad r \geq 2,
$$

is an isomorphism.

Proof. Using the dual (i.e. cochain) version of the Barr-Beck acyclic model Theorem [3], it is not hard to prove that the (cochain) maps

$$
\begin{aligned}
& E_{1} G \mathbf{Z X X} \otimes E_{1} G \mathbf{Z} X \stackrel{[,]}{\longrightarrow} E_{1} G \mathbf{Z} X \rightarrow \hat{E}^{1} X, \\
& E_{1} G \mathbf{Z} X \otimes E_{1} G \mathbf{Z} X \stackrel{\longrightarrow}{\longrightarrow} \hat{E}^{1} X \otimes \hat{E}^{1} X \stackrel{[,]}{\longrightarrow} \hat{E}^{1} X
\end{aligned}
$$

are (cochain) homotopic. This, together with 14.2, yields the first part of the theorem.

To prove the other part observe that

(i) $Q \otimes \hat{E}^{1} X$ depends functorially on $Q X$;

(ii) $Q \otimes \hat{E}^{2} X$ collapses to $\pi_{*} X$ whenever $X$ is a simplicial $Q$-module.

Hence $[7,10.7]$ we can use the arguments of $[7, \$ 10]$ to show that the map $E_{r}(X ; Q) \rightarrow Q \otimes \hat{E}^{r} X$ is an isomorphism for $r=2$ and hence for all $r \geq 2$.

15.3. The rational cobar spectral sequence. For $X \in \mathcal{S}_{*}$ connected, there are several essentially equivalent constructions for its rational cobar spectral sequence $\bar{E}^{r}(X ; Q)([1],[6],[11])$, of which we will use the one of $[6, \S 10]$ (with $Q$ instead of $\left.Z_{2}\right)$.

Recall that $\bar{E}^{r}(X ; Q)$ is actually a Hopf algebra spectral sequence (i.e. each $\left(\bar{E}^{r}, d^{r}\right)$ is a differential graded Hopf algebra). Hence, in view of [16, p. 280] and [14], the primitive elements $P \bar{E}^{r}(X ; Q)$ of $\bar{E}^{r}(X ; Q)$ yield a Lie algebra spectral sequence, and the results of $[6, \$ 10]$ then readily imply: 
15.4. Theorem. For $X \in \mathcal{S}_{*}$ connected, the natural spectral sequence map $Q \otimes \hat{E}^{r} X \rightarrow \bar{E}^{r}(X ; Q), r \geq 1$ (constructed as in $[6, \$ 10]$, with $Q$ instead of $Z_{2}$ ), induces a Lie algebra spectral sequence isomorphism $Q \otimes \hat{E}^{r} X \approx P \bar{E}^{r}(X ; Q)$, $r \geq 1$.

Combining this with 15.2 we get

15.5. Corollary. For $X \in \mathcal{S}_{*}$ connected, the natural spectral sequence map $E_{r}^{s, t+1}(X ; Q) \rightarrow Q \otimes \hat{E}_{s+1, t-s}^{r} X \rightarrow \bar{E}_{s+1, t-s}^{r}(X ; Q), \quad r \geq 2$,

induces a Lie algebra spectral sequence isomorphism $E_{r}(X ; Q) \approx P \bar{E}^{r}(X ; Q), r \geq 2$.

15.6. Corollary [15]. For $X \in \mathcal{S}_{*}$ connected, there is a natural Lie algebra isomorphism

$$
E_{2}(X ; Q) \approx P \operatorname{Cotor}^{H}(X ; Q)(Q, Q)
$$

15.7. Remark. When $H_{*}(X ; Q)$ is of finite type, then the Hopf algebra Cotor $^{H_{*}(X ; Q)}(Q, Q)$ is equivalent to the classical cohomology $E x t_{H^{*}}(X ; Q)(Q, Q)$, of the algebra $H^{*}(X ; Q)$. It is thus highly computable (see [5]).

16. The rational spectral sequence $E_{r}(W, X ; Q)$. In this section we will

(i) prove that the rational spectral sequence $E_{r}(W, X ; Q)$ is completely determined by $E_{r}(X ; Q)$ and $\widetilde{H}_{*}(W ; Q)$,

(ii) use (i) to show the essential triviality of the rational composition pairing, and

(iii) use (i) to recover a result of Arkowitz-Curjel on the rank of certain groups of bomotopy classes [2].

16.1. Reduction of $E_{r}(W, X ; Q)$. For $W, X \in \delta_{*}, X$ connected, and $t>s \geq 0$ there is a natural isomorphism

$$
E_{r}^{s, t}(W, X ; Q) \approx \prod_{n \geq 0} \widetilde{H}^{n}\left(W ; E_{r}^{s, t+n}(X ; Q)\right), \quad r \geq 1
$$

Proof. For $t \geq 1$ there is a natural isomorphism

$$
\pi_{t} \operatorname{bom}(W, \mathbf{Q} X) \approx \prod_{n \geq 0} \tilde{H}^{n}\left(W ; \pi_{t+n} \mathbf{Q} X\right)
$$

of cosimplicial $Q$-modules, which implies the cases $r=1,2$. The cases $r>2$ then follow by a straightforward induction using 10.1 and the facts

(i) if $M$ and $N$ are graded $Q$-modules, then any additive cohomology operation of the form

$$
\prod_{n \geq 0} \widetilde{H}^{n}\left(Y ; M_{n}\right) \rightarrow \prod_{n \geq 0} \widetilde{H}^{n}\left(Y ; N_{n}\right), \quad Y \in S_{*},
$$

is induced by coefficient homomorphisms $M_{n} \rightarrow N_{n}, n \geq 0$, 
(ii) for $W, X \in \mathcal{S}_{*}$ and any ring $R$ (though we only need here $R=Q$ ) there exists a natural spectral sequence $\left\{\right.$ big $\left.E_{r}(W, X ; R)\right\}$ such that

$$
\begin{aligned}
\operatorname{big} E_{2}^{s, t}(W, X ; R) & \approx \pi^{s} \pi_{t} \operatorname{bom}(W, \mathbf{R} X), & t \geq s \geq 0, & t>0, \\
& \approx 0, & & \text { otherwise, } \\
\text { big } E_{r}^{s, t}(W, X ; R) & \approx E_{r}^{s, t}(W, X ; R), & & t>s \geq 0, \quad r \geq 2 .
\end{aligned}
$$

The existence of this enlarged spectral sequence can be proved using the approach of [8]; its only usefulness is for studying $E_{r}^{s, t}(W, X ; R)$ on its "fringe" $t-s=1$ [7].

16.2. The essential triviality of the rational composition pairing. For $W, X, Y$ $\epsilon \mathfrak{S}_{*}$ with $X, Y$ connected, the composition pairing (\$9)

$$
E_{r}^{s, t}(X, Y ; Q) \otimes E_{r}^{s^{\prime}, t^{\prime}}(W, X ; Q) \stackrel{\circ}{\longrightarrow} E_{r}^{s+s^{\prime}, t+t^{\prime}}(W, Y ; Q), \quad r \geq 2
$$

is trivial if $s^{\prime}>0$.

Proof. The inclusion $\phi: X \rightarrow Q X$ induces, by 16.1 , an epimorphism $E_{r}^{s, t}(Q X, Y ; Q) \rightarrow E_{r}^{s, t}(X, Y ; Q)$ and by $[7, \S 4]$ the group $E_{r}^{s^{\prime}, t^{\prime}}(W, Q X ; Q)$ vanishes for $s^{\prime}>0$ and $r \geq 2$. The desired result now follows by a naturality argument.

16.3. Remark. The composition pairing for $s^{\prime}=0$ has an obvious description using 16.1 and the canonical inclusion $E_{r}^{0, *}(X ; Q) \subset H_{*}(X ; Q)$. The details are left to the reader.

For our second application of 16.1 we need

16.4. The rank of a group. A group $G$ is of finite rank if there exists a finite filtration

$$
G=N_{0} \supset \ldots \supset N_{i} \supset N_{i+1} \supset \ldots \supset N_{b}=1
$$

such that each $N_{i+1}$ is a normal subgroup of $N_{i}$ and each $N_{i} / N_{i+1}$ is either infinite cyclic or periodic. For $G$ of finite rank the number $\rho(G)$ of infinite cyclic $N_{i} / N_{i+1}$ is called the rank of $G$ and depends only on $G$. This notion of rank coincides with the usual one for abelian groups, and is discussed in detail in [2]. Now we can formulate

16.5. The Arkowitz-Curjel result. Let $W \in \mathcal{S}_{*}$ be finite dimensional and let $X \in \mathcal{S}_{* K}$ (3.2) be simply connected. Then the group [SW, X] (of homotopy classes rel. * of maps $S W \rightarrow X)$ bas rank

$$
\rho[S W, X]=\sum_{n>0} \rho\left(H_{n}(S W ; Z)\right) \rho\left(\pi_{n} X\right) .
$$

Proof. Combine 16.1 for $r=\infty$ with the convergence propertities of the integral spectral sequence [7] and the isomorphism [7] 


$$
E_{\infty}(W, X ; Q) \approx Q \otimes E_{\infty}(W, X ; Z)
$$

17. A homological description of the smash and composition pairings for $E_{2}\left(; Z_{p}\right)$. In [7, 11 and $\left.\$ 12\right]$ we considered the category $\mathcal{C} Q$ of (connected) unstable coalgebras over the Steenrod algebra and observed that

(i) the $Z_{p}$-bomology functor is actually a functor $H_{*}\left(; Z_{p}\right): \mathcal{S}_{*_{c}} \rightarrow \mathfrak{C Q}$ where $\mathcal{S}_{*_{c}} \subset \mathcal{S}_{*}$ is the full subcategory of connected complexes, and

(ii) for $W \in \mathcal{S}_{*}, X \in \mathcal{S}_{* c}$ and $t>s \geq 0$ there are natural isomorphisms

$$
\begin{gathered}
E_{2}^{s, t}\left(X ; Z_{p}\right) \approx \operatorname{Ext}_{\mathcal{C Q}}^{s}\left(H_{*}\left(S^{t} ; Z_{p}\right), H_{*}\left(X ; Z_{p}\right)\right), \\
E_{2}^{s, t}\left(W, X ; Z_{p}\right) \approx \operatorname{Ext}_{\mathcal{C Q}}^{s}\left(H_{*}\left(S^{t} \wedge W ; Z_{p}\right), H_{*}\left(X ; Z_{p}\right)\right)
\end{gathered}
$$

where the $\operatorname{Ext}_{\mathcal{C}}^{s}$ are, in some sense, the right derived functors of Hom Ca.

This suggests that it should be possible to give a homological description of the smash and composition pairings for $E_{2}\left(; Z_{p}\right)$, and we devote this section to showing that this indeed can be done, in fact by merely mimicking our constructions for spaces of Chapter II. But first a

17.1. Notational convention. Throughout the rest of this chapter we will freely use the notation (and results) of [7], except that from now on we will write $H_{*}$ instead of $H_{*}\left(; Z_{p}\right)$.

17.2. A smash product in $\mathcal{C} \mathscr{G}$. For $C, D \in \mathcal{C} \mathscr{A}$ let $C \wedge D \in \mathcal{C} \mathfrak{P}$ denote the quo-

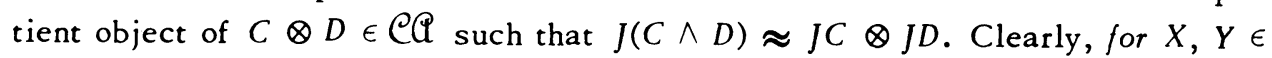
$\mathcal{S}_{* \sigma}$, there then is a natural isomorphism

$$
H_{*} X \wedge H_{*} Y \approx H_{*}(X \wedge Y) \in \mathcal{C} \mathbb{P} .
$$

17.3. The functors $E x t^{s, t}$. Let $\mathcal{C} \mathbb{Q}^{\prime} \supset \mathcal{C} \mathbb{P}$ denote the category defined in the same way as $\mathcal{C}[7, \S 11]$ but with connected replaced by co-augmented. Then, for $B \in \mathcal{C P}^{\prime}$ and $t \geq 1, H_{*} S^{t} \wedge B$ is in $\mathcal{C P}$ and has trivial comultiplication, and hence we can define functors

$$
\operatorname{Ext}_{\mathrm{C}}^{\mathrm{s}} \stackrel{\mathrm{Q}}{\mathrm{Q}}(B, \quad): \mathcal{C} \mathbb{P} \rightarrow\left(Z_{p} \text {-modules }\right)
$$

by

$$
\operatorname{Ext}_{\mathcal{C} Q \bar{Q}}^{s, t}(B,) \approx E_{x t_{C Q}^{s}}^{s}\left(H_{*} s^{t} \wedge B,\right) \quad s \geq 0, \quad t \geq 1 .
$$

As $H_{*} W \in \mathcal{C} Q^{\prime}$ for all $W \in \mathcal{S}_{*}$, the isomorphisms at the beginning of this section now can be written

$$
E_{2}^{s, t}\left(X ; Z_{p}\right) \approx E_{C} t_{C}^{s, t}\left(H_{*} S^{c}, H_{*} X\right), \quad E_{2}^{s, t}\left(W, X ; Z_{p}\right) \approx E x t_{C Q}^{s, t}\left(H_{*} W, H_{*} X\right) .
$$

17.4. The smash pairing for $E x t_{\mathcal{C}}^{s, t}\left(H_{*} S^{0}\right.$, ). For $C, D \in \mathcal{C Q P}$ let $T C \wedge T D \stackrel{a}{\rightarrow}$ $T(C \wedge D) \in \mathcal{C} \mathbb{P}$ be the adjoint of the obvious map 


$$
J(T C \wedge T D)=J T C \otimes J T D=J V J C \otimes J V J D \rightarrow J C \otimes J D=J(C \wedge D) .
$$

Then $a$ induces, as in $\$ 8$, a cosimplicial map $\mathrm{T} C \wedge \mathrm{T} D \stackrel{a}{\rightarrow} \mathrm{T}(C \wedge D)$ and we define, for $s, s^{\prime} \geq 0$ and $t, t^{\prime} \geq 1$, the smasb pairing

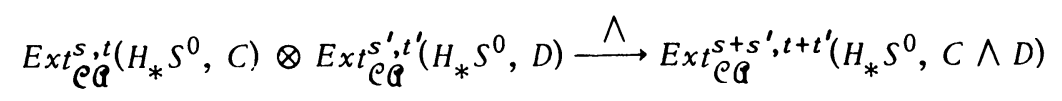

as the composition

$$
\begin{array}{r}
\pi^{s}\left[H_{*} s^{t}, \mathbf{T C}\right] \otimes \pi^{s^{\prime}}\left[H_{*} s^{t^{\prime}}, \mathbf{T} D\right] \stackrel{f}{\longrightarrow} \pi^{s+s^{\prime}}\left(\left[H_{*} s^{t}, \mathbf{T} C\right] \otimes\left[H_{*} s^{t^{\prime}}, \mathbf{T} D\right]\right) \\
\rightarrow \pi^{s+s^{\prime}}\left[H_{*} s^{t+t^{\prime}}, \mathbf{T} C \wedge \mathrm{T} D\right] \stackrel{a_{*}}{\longrightarrow} \pi^{s+s^{\prime}}\left[H_{*} s^{t+t^{\prime}}, \mathbf{T}(C \wedge D)\right]
\end{array}
$$

where [,] denotes $\mathrm{Hom}_{\mathrm{CQ}}(),$,$f is the (graded) Alexander-Whitney map (7.1)$ and the middle map is the obvious one.

Clearly this definition implies that, for $X, Y \in \mathcal{S}_{*_{c}}$ and $t>s \geq 0, t^{\prime}>s^{\prime} \geq 0$, the pairing

$$
\operatorname{Ext}_{\mathcal{C} Q}^{s, t}\left(H_{*} S^{0}, H_{*} X\right) \otimes \operatorname{Ext}_{\in \mathbb{Q}}^{s^{\prime}, t^{\prime}}\left(H_{*} s^{0}, H_{*} Y\right) \stackrel{\wedge}{\longrightarrow} \operatorname{Ext}_{\mathcal{C} Q}^{s+s^{\prime}, t+t^{\prime}}\left(H_{*} S^{0}, H_{*}(X \wedge Y)\right)
$$

coincides with the pairing ( $\$ 8)$

$$
E_{2}^{s, t}\left(X ; Z_{p}\right) \otimes E_{2}^{s^{\prime}, t^{\prime}}\left(Y ; Z_{p}\right) \stackrel{\wedge}{\longrightarrow} E_{2}^{s+s^{\prime}, t+t^{\prime}}\left(X \wedge Y ; Z_{p}\right)
$$

Similarly we deal with

17.5. The composition pairing for $E x t_{e^{s}, t}^{Q^{*}}$ For $B \in \mathcal{C Q}^{\prime}, C, D \in \mathcal{C}$ and $s$, $s^{\prime} \geq 0, t, t^{\prime} \geq 1$ we define the composition pairing

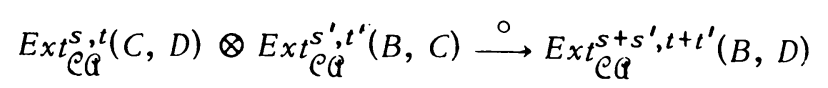

as the composite map

$\pi^{s}\left[H_{*} S^{t} \wedge C, \mathbf{T} D\right] \otimes \pi^{s^{\prime}}\left[H_{*} s^{t^{\prime}} \wedge B, \mathbf{T} C\right] \stackrel{f}{\longrightarrow} \cdots \stackrel{c_{*}}{\longrightarrow} \pi^{s+s^{\prime}}\left[H_{*} S^{t} \wedge H_{*} S^{t^{\prime}} \wedge B, \mathbf{T} D\right]$ where again $[$,$] denotes H_{C} e_{Q}($,$) and f$ is the (graded) Alexander-Whitney map, while the map

$$
\left[H_{*} S^{t} \wedge C, \mathbf{T} D\right] \otimes\left[H_{*} S^{t^{\prime}} \wedge B, \mathbf{T} C\right] \stackrel{c}{\longrightarrow}\left[H_{*} S^{t} \wedge H_{*} S^{t^{\prime}} \wedge B, \mathbf{T} D\right]
$$

is defined in the same way as the map $c$ of $\$$.

Clearly this definition also implies that for $W, X, Y \in \mathcal{S}_{*_{c}}$ and $t>s \geq 0, t^{\prime}>$ $s^{\prime} \geq 0$ the pairing

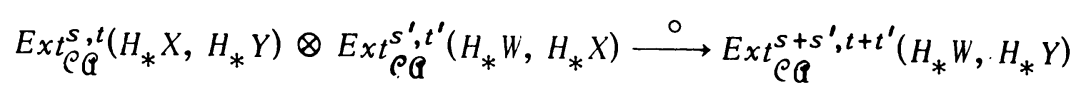

coincides with the pairing (\$9)

$$
E_{2}^{s, t}\left(X, Y ; Z_{p}\right) \otimes E_{2}^{s^{\prime}, t^{\prime}}\left(W, X ; Z_{b}\right) \stackrel{\circ}{\longrightarrow} E_{2}^{s+s^{\prime}, t+t^{\prime}}\left(W, Y ; Z_{p}\right)
$$


18. The composition action of $E_{2}\left(S^{m} ; Z_{p}\right)$ in the Massey-Peters on case. We now combine 17.5 with the results of $[7, \$ 13]$ to give a useful simple description of the composition action of $E_{2}\left(S^{m} ; Z_{p}\right)$ on $E_{2}\left(X ; Z_{p}\right)$ for "very nice" $X$ in terms of the classical Yoneda product.

18.1. The description in terms of the Yoneda product. For $X \in \mathscr{S}_{*_{c}}$ and $t>$ $s \geq 0, t^{\prime}>s^{\prime} \geq 0$, the composition action $(\$ 10)$

$$
E_{2}^{s, t+m}\left(X ; Z_{p}\right) \otimes E_{2}^{s^{\prime}, t^{\prime}}\left(S^{m}, Z_{p}\right) \stackrel{\circ}{\longrightarrow} E_{2}^{s+s^{\prime}, t+t^{\prime}}\left(X ; Z_{p}\right)
$$

corresponds, by 17.5 to the composition

(ii) $\operatorname{Ext}_{\mathrm{C}}^{s, t}\left(H_{*} s^{m}, H_{*} X\right) \otimes \operatorname{Ext}_{\mathrm{C} Q}^{s^{\prime}, t^{\prime}}\left(H_{*} s^{0}, H_{*} s^{m}\right) \stackrel{\circ}{\longrightarrow} \operatorname{Ext}_{\mathrm{C}}^{s+s^{\prime}, t+t^{\prime}}\left(H_{*} s^{0}, H_{*} X\right)$

But, if $X$ and $S^{m}$ are "very nice" (i.e. if there is an $M \in \mathbb{M Q}$ such that $H_{*} X \approx U M \in \mathcal{C}(\dot{H}$ and either $m$ is odd or $p=2$ ), then $H_{*} S^{m}=U \tilde{H}_{*} S^{m}$ and hence $[7,13.6]$ the composition

(ii) corresponds to a composition

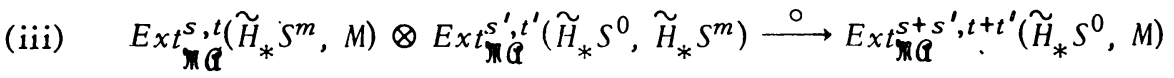
where the Ext $t^{s, t}$ are defined in terms of the Ext $t_{M Q}^{s}$ in the same manner as the $E x t_{C Q}^{s, t}$ were defined in terms of the Ext $e_{Q Q}^{s}$ (17.3) and the composition pairing $\circ$ for the $E x t_{M}^{s, t}$ is constructed as the one for the $E x t_{C}^{s, t}(17.5)$ (using the functors $J^{\prime \prime}$ and $V^{\prime \prime}$ instead of $J$ and $V$ ). Finally, as $M Q$ is an abelian category we can (and will) identify $E x t_{\text {Ma }}^{s}$ with the Yoneda group of $s$-fold extensions using the correspondence $\breve{\zeta}$ of $[12$, p. 96$]$ and a straightforward calculation then yields that the composition (iii) corresponds to $(-1)^{s s^{\prime}+t s^{\prime}}$ times the composite map

$$
\operatorname{Ext}_{\Re \mathbb{Q}}^{s}\left(\widetilde{H}_{*} s^{t+m}, M\right) \otimes \operatorname{Ext}_{\Re Q}^{s^{\prime}}\left(\tilde{H}_{*} s^{t^{\prime}}, \widetilde{H}_{*} s^{m}\right)
$$

$$
\rightarrow \operatorname{Ext}_{M Q}^{s}\left(\widetilde{H}_{*} s^{t+m}, M\right) \otimes E x t_{M Q}^{s^{\prime}}\left(\widetilde{H}_{*} s^{t+t^{\prime}}, \widetilde{H}_{*} s^{t+m}\right) \rightarrow E x t_{M Q}^{s+s^{\prime}}\left(\widetilde{H}_{*} s^{t+t^{\prime}}, M\right)
$$

where the first map is induced by the operation $\widetilde{H}_{*} S^{t} \otimes-$ and the second map is the Yoneda product $[12$, p. 82$]$ in the abelian category $M \mathbb{Q}$.

19. A homological description of the Whitehead product in $E_{2}\left(; Z_{p}\right)$. Using our second construction for the Whitehead product in $E_{1}$ (13.4) we will

(i) show that the Whitehead product in $E_{2}\left(; Z_{p}\right)$ corresponds to a certain homological product in Ext $\mathrm{CQ}\left(H_{*} S^{0}\right.$, ), and

(ii) use this to show that the Whitehead product in $E_{2}\left(; Z_{p}\right)$ (and hence in $E_{r}\left(; Z_{p}\right)$ for $\left.r \geq 2\right)$ vanishes for "very nice" spaces (i.e. in the Massey-Peterson case).

19.1. The homological Whitehead product. For $C \in \mathcal{C Q}$ and $s, s^{\prime} \geq 0, t, t^{\prime} \geq$ 1 , we define the homological Whitebead product

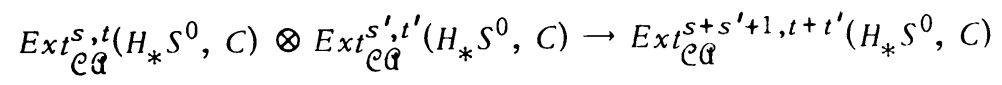

as the composite map 


$$
\begin{gathered}
\pi^{s}\left[H_{*} s^{t}, \mathbf{T} C\right] \otimes \pi^{s^{\prime}}\left[H_{*} s^{t^{\prime}}, \mathbf{T} C\right] \stackrel{f}{\longrightarrow} \pi^{s+s^{\prime}}\left(\left[H_{*} s^{t}, \mathbf{T} C\right] \otimes\left[H_{*} s^{t^{\prime}}, \mathbf{T C}\right]\right) \\
\rightarrow \pi^{s+s^{\prime}}\left[H_{*} s^{t+t^{\prime}}, \mathbf{T} C \wedge \mathbf{T} C\right] \stackrel{w_{*}}{\longrightarrow} \pi^{s+s^{\prime}+1}\left[H_{*} s^{t+t^{\prime}}, \mathbf{T} C\right]
\end{gathered}
$$

where again [,] stands for $\operatorname{Hom}_{\mathrm{CQ}}(),$,$f is the (graded) Alexander-Whitney map$ (7.1) and the middle map is the obvious one, while $w_{*}$ is induced by the composite maps

$$
T^{k} C \wedge T^{k} C \stackrel{\zeta}{\longrightarrow} T\left(T^{k} C \otimes T^{k} C\right) \stackrel{T(\times)}{\longrightarrow} T^{k+1} C, \quad k \geq 1,
$$

where $\zeta$ is the adjoint of the obvious inclusion

$$
J\left(T^{k} C \wedge T^{k} C\right)=J T^{k} C \oplus J T^{k} C \rightarrow J\left(T^{k} C \otimes T^{k} C\right)
$$

and $\times: T^{k} C \otimes T^{k} C \rightarrow T^{k} C$ is the "multiplication map"

$T^{k} C \otimes T^{k} C=V J T^{k-1} C \otimes V J T^{k-1} C=V\left(J T^{k-1} C \oplus J T^{k-1} C\right) \stackrel{V(+)}{\longrightarrow} V J T^{k-1} C=T^{k} C$

induced by the "addition map" +: J $T^{k-1} C \oplus J T^{k-1} C \rightarrow J T^{k-1} C$. A lengthy but straightforward calculation shows that $w_{*}$ is well defined, and it then follows readily from 13.5 that, for $X \in \mathcal{S}_{*_{c}}$ and $t>s \geq 0, t^{\prime}>s^{\prime} \geq 0$, the bomological Whitebead product

$$
E_{\mathcal{C Q}} t^{s, t}\left(H_{*} s^{0}, H_{*} X\right) \otimes E x t_{\mathcal{C}}^{s^{\prime}, t^{\prime}}\left(H_{*} s^{0}, H_{*} X\right) \rightarrow E x t_{\mathcal{C Q}}^{s+s^{\prime}+1, t+t^{\prime}}\left(H_{*} s^{0}, H_{*} X\right)
$$

corresponds to $(-1)^{t-s-1}$ times the Whitebead product $(\$ 14)$

$$
E_{2}^{s, t}\left(X ; Z_{p}\right) \otimes E_{2}^{s^{\prime}, t^{\prime}}\left(X ; Z_{p}\right) \stackrel{[,]}{\longrightarrow} E_{2}^{s+s^{\prime}+1, t+t^{\prime}}\left(X ; Z_{p}\right) .
$$

19.2. The Massey-Peterson case. If $X \in \mathcal{S}_{*_{c}}$ is "very nice" (i.e. there is an $M \in \mathbb{M}$ such that $\left.H_{*} X \approx U M \in \mathcal{C}\right)$, then the Whitebead product in $E_{r}\left(X ; Z_{p}\right)$ is trivial for $2 \leq r \leq \infty$.

This follows readily from 19.1 , the fact that $U M \in \mathcal{C Q}$ is an "H-object" (i.e.

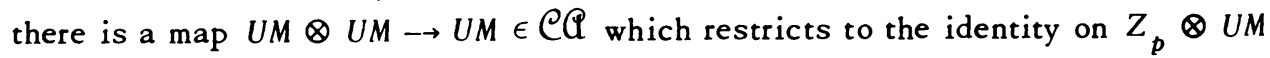
and $\left.U M \otimes Z_{p}\right)$, and the following

19.3. Lemma. If $C \in \mathcal{C Q}$ is an H-object, then the bomological Whitebead product in $\operatorname{Ext}_{\mathrm{CQ}}\left(\mathrm{H}_{*} \mathrm{~S}^{0}, \mathrm{C}\right)$ is trivial.

For the proof of this lemma (which is similar to the proof that in an $H$-space all Whitehead products are trivial) one needs

19.4. Proposition. For $C, D \in \mathcal{C Q}$ and $s \geq 0, t \geq 1$, there is a natural isomorphism

$$
\operatorname{Ext}_{\mathcal{C} Q}^{s, t}\left(H_{*} s^{0}, C \otimes D\right) \approx E_{\mathcal{C}}^{s, t}\left(H_{*} s^{0}, C\right) \oplus \operatorname{Ext}_{\mathrm{C}}^{s, t}\left(H_{*} s^{0}, D\right) .
$$

This follows from $[7,12.2]$ using the natural isomorphism 


$$
H_{C Q}\left(H_{*} S^{t}, \mathbf{T} C \otimes \mathbf{T} D\right) \approx H_{C} m_{e}\left(H_{*} S^{t}, \mathbf{T} C\right) \oplus H_{C Q}\left(H_{*} s^{t}, \mathbf{T} D\right)
$$

and the fact that $\mathrm{T} C \otimes \mathrm{T} D$ is a cosimplicial resolution of $C \otimes D$.

19.5. Remark. Another interesting consequence of 19.4 is the fact that, for $X, Y \in \mathcal{S}_{* c}$, there is a natural isomorphism

$$
E_{2}\left(X \times Y ; Z_{p}\right) \approx E_{2}\left(X ; Z_{p}\right) \oplus E_{2}\left(Y ; Z_{p}\right)
$$

\section{BIBLIOGRAPHY}

1. J. F. Adams, On the cobar constructioı, Colloq. de Topologie Algébrique (Louvain, 1956), Georges Thone, Liège; Masson, Paris, 1957, pp. 81-87. MR 19, 759.

2. M. Arkowitz and C. R. Curjel, Groups of homotopy classes. (Rank formulas and homotopy-commutativity), Lecture Notes in Math., vol. 4, Springer-Verlag, Berlin and New York, 1964. MR $32 \# 1702$.

3. M. Barr and J. Beck, Acyclic models and triples, Proc. Conf. Categorical Algebra (La Jolla, Calif., 1965), Springer, New York, 1966, pp. 336-343. MR 39 \#6955.

4. J. M. Boardman and B. Steer, On Hopf invariants, Comment. Math. Helv. 42 (1967), 180-221. MR $36 \# 4555$.

5. A. K. Bousfield, Nice homology coalgebras, Trans. Amer. Math. Soc. 148 (1970), 473-489. MR $41 \# 3564$.

6. A. K. Bousfield and E. B. Curtis, A spectral sequence for the homotopy of nice spaces, Trans. Amer. Math. Soc. 151 (1970), 457-479. MR $42 \# 2488$.

7. A. K. Bousfield and D. M. Kan, The homotopy spectral sequence of a space with coefficients in a ring, Topology 11 (1972), 79-106.

8. - A second quadrant homotopy spectral sequence, Trans. Amer. Math. Soc. 177 (1973), 305-318.

9. E. B. Curtis, Simplicial homotopy the ory, Advances in Math 6 (1971), 107-209. MR $43 \# 5529$.

10. S. Eilenberg and J. C. Moore, Adjoint functors and triples, Illinois J. Math. 9 (1965), 381-398. MR $32 \# 2455$.

11. S. Eilenberg and J. C. Moore, Homology and fibrations. I. Coalgebras, cotensor product and its derived functors, Comment. Math. Helv. 40 (1966), 199-236. MR 34 \#3579.

12. S. Mac Lane, Homology, Die Grundlehren der math. Wissenschaften, Band 114, Academic Press, New York; Springer-Verlag, Berlin, 1963. MR 28 \#122.

13. J. P. May, Simplicial objects in algebraic topology, Van Nostrand Math. Studies, no. 11, Van Nostrand, Princeton, N. J., 1967. MR 36 \#5942.

14. J. W. Milnor and J.C. Moore, On the structure of Hopf algebras, Ann. of Math. (2) 81 (1965), 211-264. MR 30 \#4259.

15. J. C. Moore and L. Smith, Hopf algebras and multiplication fibrations. II, Amer. J. Math. 90 (1968), 1113-1150。 MR 38 \#6599.

16. D. G. Quillen, Rational homotopy theory, Ann。 of Math. (2) 90 (1969), 205-295. MR $41 \# 2678$. 
17. D. L. Rector, Steenrod operations in the Eilenberg-Moore spectral sequence, Comment. Math. Helv. 45 (1970), 540-552.

DEPARTMENT OF MATHEMATICS, BRANDEIS UNIVERSITY, WALTHAM, MASSACHUSETTS 02154

DEPAR TMENT OF MATHEMATICS, MASSACHUSETTS INSTITUTE OF TECHNOLOGY, CAMBRIDGE, MASSACHUSETTS 02139 (Current address of D. M. Kan)

Current address (A. K. Bousfield): Department of Mathematics, University of Illinois at Chicago Circle, Chicago, Illinois 60680 